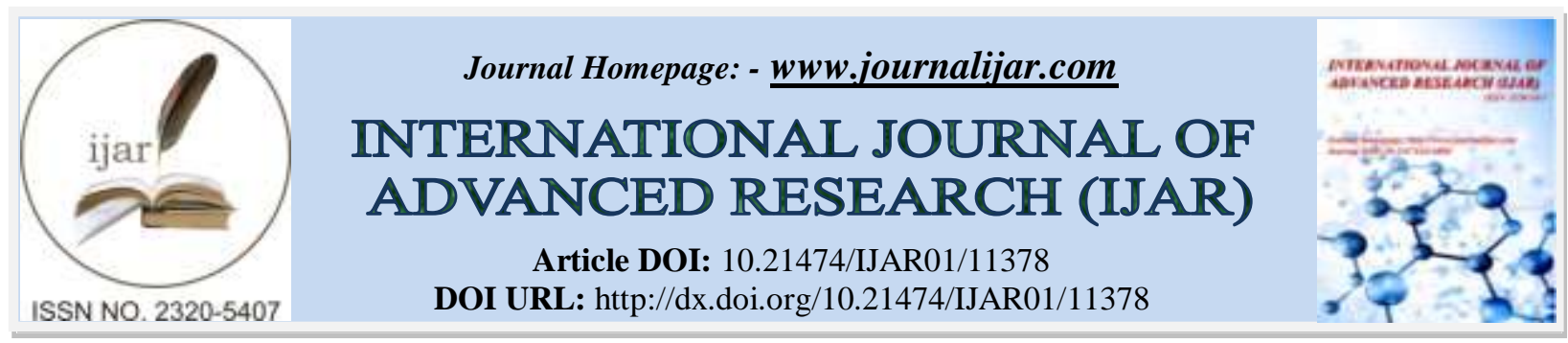

RESEARCH ARTICLE

\title{
PHASE SEPARATION OF BONDING AGENTS
}

\section{Dr. Prashanth Kumar Katta MDS ${ }^{1}$ and Dr. Sreedhara Shantharam BDS, MDS ${ }^{2}$}

1. Assistant professor, Department Of Restorative Dental Sciences, College of Dentistry, King Faisal University, Al Hasa, Kingdom Of Saudi Arabia.

2. Specialist Orthodontist, Najran Specialty Dental Center, Ministry of Health, Najran, Kingdom of Saudi Arabia.

\section{Manuscript Info}

[.......................

Manuscript History

Received: 20 May 2020

Final Accepted: 24 June 2020

Published: July 2020

Key words:-

Adhesive, Dentin Interface, Hydrophilic-

Rich Phase, Phase Separation

\section{Abstract}

Dentin bonding agents have changed significantly with time from multi bottle to single bottle. The bonding agent plays a crucial role in the long term survival of the composite restoration. Also, the composition of bonding agents has changed to incorporate self-etching agents into the bonding agents to avoid a separate etching step. This article highlights the phase separation and changes in bonding agents with time and how it will affect the bonding mechanism.

Copy Right, IJAR, 2020,. All rights reserved.

\section{Introduction:-}

Even though there are advances in bonding systems the bonding to tooth structure is a challenge. Formation of the hybrid layer by resin penetration into the demineralized dentin is very important. The hybrid layer is a highly organic interface that is relatively hydrophobic, acid resistant, and tough Phase separation into hydrophobic-rich and hydrophilic-rich phases during infiltration of the over-wet demineralized collagen matrix. Such phase separation undermines the integrity and durability of the bond at the composite/tooth interface ${ }^{1,2}$.

In the wet bonding technique, the demineralized collagen matrix remains excessively wet. Consequentially, the current dentin adhesives go through physical separation during penetration into hydrophobic- and hydrophilic-rich phases

The new generation dentin adhesives are sensitive to moisture, seen as nanoleakage in the hybrid layer and phase separation into hydrophobic- and hydrophilic-rich phases. A typical finding in HEMA-free materials is caused by the incompatiblity of hydrophobic and amphiphilic monomers, upon solvent evaporation. Nano-phase separation decreases the dynamic mechanical properties of the polymerized adhesives and increases their susceptibility to esterase-catalyzed hydrolysis ${ }^{1,3}$.

During acid etching the mineral phase is extracted from a zone that measures between 1 and $\sim 10$ micrometer of the dentin surface.

Adhesives are compounds containing both hydrophilic and hydrophobic monomers. The major difference between hydrophilic and hydrophobic adhesives is the chemistry of their monomers and solvents. Adhesive systems are designed to promote dentin adhesion through the interaction of hydrophilic monomers in an organic solvent throughout a collagen rich humid tissue ${ }^{2,4}$. 
The earlier generation were of etch and rinse type of bonding agents. The new generation bonding agents are selfetch bonding agents which don't need a separate etching step. All the three component etchant, primer and adhesive are in a single bottle.

The hydrophilic component shows minimal monomer/polymer conversion because of adhesive phase separation and lack of compatibility between the hydrophobic photoinitiator and hydrophilic phase. This contributes to the degradation of hybrid layer over a period of time ${ }^{2,6}$.

As the adhesive disperses through the wet, demineralized dentin matrix, it passes through a phase separation into hydrophobic and hydrophilic-rich phases. Water will spread into the loosely cross-linked or hydrophilic components. Degradation due to water can break the bonds within the adhesive and may undermine the dentin/adhesive bond integrity ${ }^{3,7}$.

resulting in the formation of blister- and globule-like voids at the resin dentine interface Furthermore, excessive moisture on the adherent substrate is also responsible for a lower degree of resin monomer conversion, reducing the mechanical properties of the adhesive layer. Most methacrylates are hydrophobic and insoluble in water, which is why many manufacturers use ethanol as a solvent to ensure that they remain in solution in one phase. The application of these adhesives in water saturated dentin after acid conditioning, generates separation in nanophases of adhesives ${ }^{4,7}$

\section{Phases in bonding agents:}

Hydrophilic primer and a hydrophobic bonding resin. The hydrophobic-rich phase is viscous due to the substantial concentration of BisGMA. The composition of the hydrophilic-rich phase is primarily water and HEMA.

The hydrophobic components of the dental adhesive (BisGMA, CQ and ethyl-4-(dimethylamino) benzoate (EDMAB) are present in least quantity in the hydrophilic-rich phase. The solvent in bonding agents is ethanol, and it displaces water on the wet demineralized surface of tooth, but even with these solvents complete removal of water is not possible and promotes detrimental adhesive phase separation ${ }^{3,8,9}$.

Under in vivo conditions, there is minimal control on the amount of water remaining on the tooth surface. There is the risk of leaving the dentin surface so wet that the adhesive actually undergoes physical separation into hydrophobic and hydrophilic-rich phases. The hydrophilic elements penetrate the interior of the hybrid layer, while the hydrophobic monomers remain on the surface. Being hydrophobic, the camphorquinones do not penetrate, leading to inadequate polymerization in the deepest zone of the hybrid layer. Problems associated with phase separation affected fundamental properties like degree of conversion and hardness, with a negative impact on interfacial strength ${ }^{5,6}$.

Phase separation of the adhesive that permeates the demineralized dentin matrix would undermine the structural integrity of the resultant hybrid layer. Adhesive phase separation can cause a very porous hybrid layer characterized by hydrophobic BisGMA-rich particles distributed in a hydrophilic HEMA-rich matrix ${ }^{4,8}$.

\section{HEMA in bonding agents:}

Conventional adhesives usually contain HEMA in a concentration between 35 and $55 \mathrm{vol} \%$. A primer containing specific monomers with hydrophilic properties, such as 2-Hydroxy ethyl methacrylate (HEMA), dissolved in organic solvents like acetone, ethanol or water. It is a low molecular weight water-soluble monomer that improves the miscibility and solubility of the polar and non-polar adhesive components. While HEMA function is to improve the wettability and promoting the re-expansion of the collagen network, the solvents are able to displace water from the dentine surface, thus make the collagen network ready for the infiltration by the following adhesive resin. The absence of HEMA makes the adhesive more prone to the possibility of the so-called phenomenon of phase separation. Water is a good solvent for HEMA but a nonsolvent for BisGMA. Systems without HEMA undergo phase separation after solvent evaporation (ethanol/acetone) and droplets of water gradually rise to the surface and can be retained if adhesive polymerization starts immediately. Clinically, a strong air-drying step is highly recommended in order to blow the droplets out, leaving only a thin layer of bonding $\operatorname{resin}^{8,10,11,12}$.

Regarding solvent, no difference in bonding effectiveness was found between the ethanol/water and acetone/water combinations, though the latter is preferred, since both functional monomers appeared more stable in acetone/water. 
Once a certain amount of acetone evaporates, the water in the bonding material separates; that is, they are phaseseparation bonding materials. HEMA-containing adhesives are more prone to water contamination, as HEMA in the uncured adhesive may absorb water which can lead to dilution of monomers to the extent that polymerization is inhibited $^{9,13,14}$.

After polymerization, HEMA will exhibit hydrophilic properties and consequentially cause water uptake and will lead to swelling and discoloration, as it is fixed in polymer chain for longer time. It also negatively affects the mechanical strength. Furthermore, high amounts of HEMA will cause flexible polymers with poor quality. HEMA has also been shown to retain water in the adhesive layer, which could reduce polymerization.

Dental adhesive compositions are primarily based on volatile diluents that cause adhesive phase separation during the last stages of flow as solvent loss is occurring rapidly Adhesive systems containing hydrophilic primers dissolved in acetone were found to produce higher bond strengths when acid-conditioned dentine was left visibly moist prior to bonding, a protocol commonly referred to as the 'wet bonding technique. Acetone has a so-called "water-chasing" effect ${ }^{11,15}$.

Hydrophilicity, however, is a both advantages and disadvantages, as, for example, HEMA absorbs water both in the cured and uncured states, which may hinder polymerization, weaken mechanical properties, and cause hydrolytic degradation of polymerized adhesive $\mathrm{e}^{13,14,16}$.

\section{Conclusion:-}

Problems related to phase separation adversely affected fundamental properties like degree of conversion and hardness, with a negative impact on interfacial strength. As the wetness of bonded surfaces varies with dentin structure, that is completely normal as compared to caries-affected, dentin depth and the presence of remaining rinse water, it is essential to understand the effect of water content on polymer formation. The composition of bonding agents should be known and proper technique should be followed for bonding procedures.

\section{Conflict of interest:}

None

\section{Source of funding:}

Self

\section{Acknowledgements:-}

None

\section{Ethical clearance:}

Not applicable

\section{References:-}

1. Classification review of dental adhesive systems: from the IV generation to the universal type. Sofan E1, Sofan A1, Palaia G1, Tenore G1, Romeo U1, Migliau G1. Ann Stomatol (Roma). 2017 Jul 3;8(1):1-17

2. Dentin Bonding: Can We Make it Last? L Tja“derhane. Oper Dent. 2015 Jan-Feb;40(1):4-18. 4-18

3. Monomer-solvent phase separation in one-step self-etch adhesive. Van Landuyt KL ${ }^{1}$, De Munck J, Snauwaert J, Coutinho E, Poitevin A, Yoshida Y, Inoue S, Peumans M, Suzuki K, Lambrechts P, Van Meerbeek B. J Dent Res. 2005 Feb;84(2):183-8.

4. Polymerization Behavior of HydrophilicRich Phase of Dentin Adhesive F. Abedin1,2, Q. Ye1 , R. Parthasarathy1 , A. Misra1,3, and P. Spencer, Journal of Dental Research 2015, Vol. 94(3) 500-507

5. Effect of simplified ethanol-wet bonding on microtensile bond strengths of dentin adhesive agents with different solvents Muhammet KerimAyar Journal of Dental Sciences Volume 10, Issue 3, September 2015, Pages 270274

6. Effect of Water on Bonding of One-bottle Self-etching Adhesives, Hashimoto M ${ }^{1}$, Fujita S, Kaga M, Yawaka Y. Dent Mater J. 2008 Mar;27(2):172-8.

7. Glutaraldehyde/HEMA in Clinical Dentistry. Leendert (Len) Boksman, DDS, Dent Today. 2011 Sep;30(9):60 
8. Resin-Dentin Bonding Interface: Mechanisms of Degradation and Strategies for Stabilization of the Hybrid Layer. DE Betancourt P. A. Baldion , and J. E. Castellano, International Journal of Biomaterials 2019(3):1-11

9. Adhesive phase separation at the dentin interface under wet bonding conditions Paulette Spencer, Yong Wan, Volume62, Issue3

10. Adhesives: Newer Is Not Always Better-Part 2, Leendert (Len) Boksman, DDS, BSc, FADI, FICD | Gregg Tousignant, CDT | Lee W. Boushell, DMD, MS | Gildo Coelho Santos, Jr., DDS, MSc, PhD, Inside Dentistry March 2012 Volume 8, Issue 3

11. Current aspects on bonding effectiveness and stability in adhesive dentistry, MV Cardoso, ${ }^{*}$ A de Almeida Neves,* A Mine,* E Coutinho,* K Van Landuyt,* J De Munck,B Van Meerbeek, Australian Dental Journal 2011; 56:(1 Suppl): 31-44

12. A review of adhesion science, 1 Marshall SJ ${ }^{1}$, Bayne SC, Baier R, Tomsia AP, Marshall GW. Dent Mater. 2010 Feb;26(2):e11-6.

13. Nanophase Separation of Polymers Exposed to Simulated Bonding Conditions Qiang Ye1,2, Yong Wang3, and Paulette Spencer, J Biomed Mater Res B Appl Biomater. 2009 February ; 88(2): 339-348.

14. Polymerization- and Solvent-Induced Phase Separation in Hydrophilic-rich Dentin Adhesive Mimic, Farhana Abedin1,2, Qiang Ye1, Holly J Good1, Ranganathan Parthasarathy1, and Paulette Spencer, Acta Biomater. 2014 July ; 10(7): 3038-3047.

15. Dentinal variations after application of an adhesive containing isopropanol used in etch and rinse mode on moist and dried dentin Geneviève Grégoire1*, Patrick Sharrock2 and Bruno Tavernier, Volume 4: 1-5, Oral Health Care, 2019

16. New strategy to create "Super Dentin" using adhesive technology: Reinforcement of adhesive-dentin interface and protection of tooth structures, ToruNikaido ${ }^{a}$ GoInoue ${ }^{\mathrm{a}}$ TomohiroTakagaki ${ }^{\mathrm{a}}$ KanchanaWaidyasekera $^{\mathrm{a}}$ YasuhiroIida ${ }^{\mathrm{a}}$ Mirela S.Shinohara ${ }^{b c}$ AlirezaSadr JunjiTagami, Japanese Dental Science Review Volume 47, Issue 1, February 2011, Pages 31-42. 